\title{
L'échographie mobile pour diagnostiquer la pneumonie
}

\author{
Alejandro Morales-Ortega MD, Jesús Canora-Lebrato PhD, José Manuel Ruiz-Giardín PhD
}

Citation : CMAJ 2021 September 20;193:E1463-4. doi : 10.1503/cmaj.210038-f

Voir la version anglaise de l'article ici : www.cmaj.ca/lookup/doi/10.1503/cmaj.210038
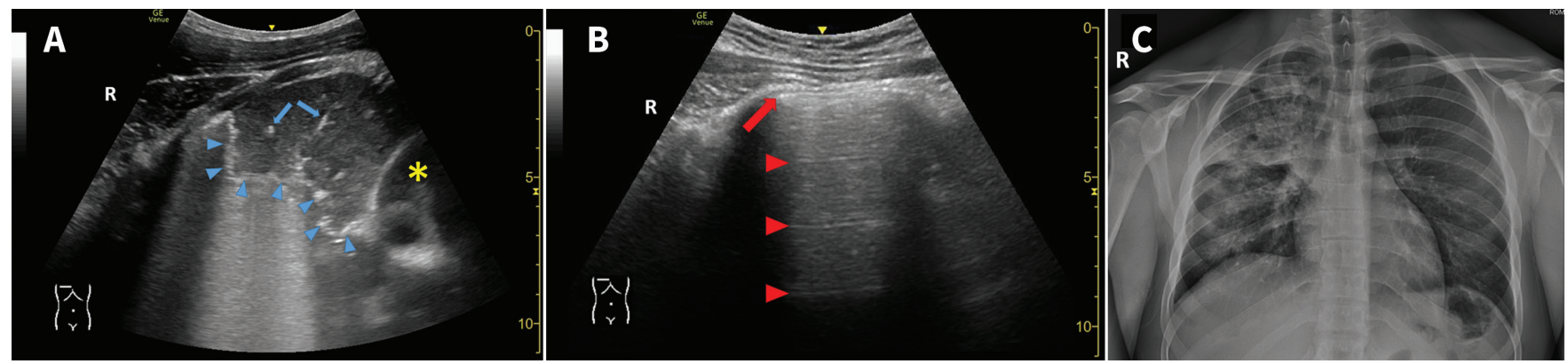

Figure 1 : A) Échogramme en coupe intercostale antérieure transversale (obtenue à l'aide d'une sonde convexe de 1 à $5 \mathrm{MHz}$ ) montrant une pneumonie, vue comme une zone hépatisée qui se comporte à la manière d'une fenêtre acoustique permettant la visualisation du cœur (astérisque). Une ligne irrégulière et hyperéchogène (pointes de flèches) accompagnée d'artefacts sonores représente le contour profond de la consolidation. Les bronchogrammes aériens (flèches) se manifestent comme des structures hyperéchogènes au sein de la consolidation pulmonaire; leur mouvement, entraîné par la respiration, peut être observé dans la vidéo (annexe 1, accessible en anglais au www.cmaj.ca/lookup/doi/10.1503/cmaj.210038/tab-related -content). B) Échogramme d'un poumon sain, obtenu avec la même sonde. Le parenchyme pulmonaire n'est pas visible puisque la plupart des ondes ultrasonores sont réfléchies sur la plèvre, qui se manifeste comme une ligne hyperéchogène (flèche), entraînant la formation d'artefacts horizontaux et répétitifs nommés « ligne $\mathrm{A}$ » (pointes de flèches). C) Radiographie du thorax montrant la consolidation des lobes supérieurs et moyens droits.

$\mathbf{U}$ n homme de 25 ans s'est présenté au service des urgences avec des symptômes de fièvre et de toux depuis 7 jours. Au moment de son hospitalisation, sa tension artérielle et son pouls étaient normaux; cependant, sa fréquence respiratoire était de 24 respirations par minute et sa saturation en oxygène à l'air ambiant était de $94 \%$. Nous entendions des bruits de respiration diminués et des crépitements inspiratoires à l'auscultation du côté supérieur droit du thorax. Les résultats d'analyses révélaient des niveaux élevés de protéines $C$ réactives et une numération leucocytaire dans les limites de la plage normale. Nous avons procédé à une échographie mobile (figure $1 \mathrm{~A}$ ) qui montrait une consolidation bien définie de forme triangulaire, accompagnée d'une échotexture s'apparentant aux tissus des lobes pulmonaires supérieur et moyen droits (c.-à-d., des poumons hépatisés), suggérant une pneumonie. La transition entre les zones consolidées et celles normalement aérées apparaissait comme une ligne hyperéchogène irrégulière (signe déchiqueté ou fractal) ${ }^{1}$. Nous avons observé un bronchogramme aérien dynamique (c.-à-d., la présence d'air dans les bronches, révélée par des structures hyperéchogènes ramifiées au sein du poumon consolidé qui se déplacent avec la respiration), un autre signe évocateur d'une pneumonie. Nous avons réalisé une radiographie pulmonaire qui a confirmé une consolidation importante du poumon droit (figure 1C). Nous avons hospitalisé le patient et avons traité sa pneumonie de façon empirique à l'aide d'une bithérapie composée de céphalosporines de troisième génération et de clarithromycine. Nous n'avons pas été en mesure de cibler l'agent pathogène et avons accordé son congé au patient après une amélioration clinique d'une période de 6 jours.

Les artefacts échographiques observés en échographie pulmonaire offrent de précieux renseignements sur le parenchyme pulmonaire qui pourraient être sous-estimés sur des radiographies thoraciques ${ }^{1,2}$. On peut assez facilement reconnaître des pneumonies importantes présentant les caractéristiques observées dans le cas présent ${ }^{3}$. Des symptômes plus subtils, comme un glissement pleural unilatéral aboli accompagné de lignes de respiration et de lignes focales B (c.-à-d., des lignes verticales hyperéchogènes découlant de la plèvre), peuvent aider à cibler les consolidations de plus petites dimensions dans certains contextes cliniques ${ }^{1,2}$. Par ailleurs, l'échographie peut aussi révéler des complications, comme une effusion pleurale ${ }^{1-3}$. 
Bien que l'expertise de l'opérateur de l'appareil puisse être limitée ${ }^{3}$, l'échographie mobile du poumon peut représenter un examen d'imagerie initial pour les cas soupçonnés de pneumonie dans les établissements hospitaliers et ambulatoires, étant donné la précision diagnostique qu'elle offre (une sensibilité et une spécificité générales de $85 \%-95 \%$ et de $75 \%-90 \%$, respectivement) ${ }^{3}$, son processus d'apprentissage rapide, son aspect sécuritaire et mobile, son faible coût relatif et sa disponibilité à grande échelle.

\section{Références}

1. Lichtenstein DA. Lung ultrasound in the critically ill. Ann Intensive Care 2014;4:1.

2. Nazerian P, Volpicelli G, Vanni S, et al. Accuracy of lung ultrasound for the diagnosis of consolidations when compared to chest computed tomography. Am J Emerg Med 2015;33:620-5.

3. Staub LJ, Mazzali Biscaro RR, Kaszubowski E, et al. Lung ultrasound for the emergency diagnosis of pneumonia, acute heart failure, and exacerbations of chronic obstructive pulmonary disease/asthma in adults: a systematic review and meta-analysis. J Emerg Med 2019;56:53-69.

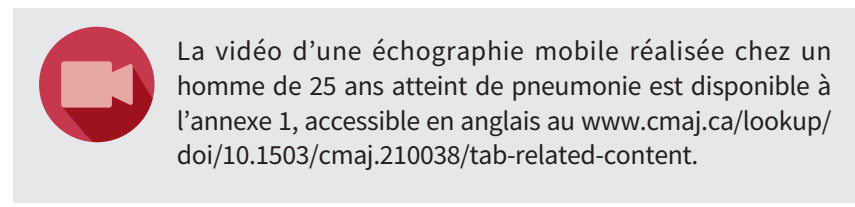

Intérêts concurrents : Aucun déclaré.

Cet article a été révisé par des pairs.

Les auteurs ont obtenu le consentement du patient.

Affiliations : Service de médecine interne, Hôpital universitaire de Fuenlabrada, Madrid, Espagne.

Propriété intellectuelle du contenu : Il s'agit d'un article en libre accès distribué conformément aux modalités de la licence Creative Commons Attribution (CC BY-NC-ND 4.0), qui permet l'utilisation, la diffusion et la reproduction de tout médium à la condition que la publication originale soit adéquatement citée, que l'utilisation se fasse à des fins non commerciales (c.-à-d., recherche ou éducation) et qu'aucune modification ni adaptation n'y soit apportée. Voir : https://creativecommons.org/licenses/by-nc-nd/4.0/deed.fr.

Correspondance : Alejandro Morales Ortega, alejandro.morales@salud.madrid.org

Les images cliniques sont choisies pour leur caractère particulièrement intéressant, classique ou impressionnant. Toute soumission d'image de haute résolution claire et bien identifiée doit être accompagnée d'une légende aux fins de publication. On demande aussi une brève explication (300 mots maximum) de la portée éducative des images, et des références minimales. Le consentement écrit du patient au regard de la publication doit être obtenu avant la soumission. 